\title{
ASTRONOMY
}

\section{A constant surprise}

\section{Whether ancient or new, in distant galaxies or our own cosmic back-yard, stars have dramatic similarities that hint at remarkably robust formative processes.}

\section{John Cowan}

The Big Bang gave us hydrogen, helium and a fraction of lithium. All the other elements in nature - the iron in our blood, the calcium in our bones and the gold in our jewelry - were synthesized inside stars that lived and died millions or even billions of years ago. In other words, the nature and extent of the synthesis of elements over the history of the Universe has changed with time and stellar evolution. Yet one aspect of stars has altered very little over billions of years, and of light years: the relative abundance patterns of certain heavy elements. These are consistent all the way through, from the Methuselahs of the star world to infants such as our Sun.

This suggests that the conditions forming these elements within stars have remained unchanged since the early Universe. Otherwise, today's stars would be producing a cocktail of elements different from the ancient stellar recipe. These striking similarities could help us to explore the nature of element formation throughout the Universe.

These findings answer a fundamental question of today's large surveys and detailed observations of the oldest surviving stars in our Galaxy and beyond. That is, whether the processes and stars that formed elements in our Galaxy were unique, or part of a broader pattern spanning large distances and times. Is our 'local neighbourhood' special, or really rather similar to other parts of the Universe?

Over the past decade, astronomical observations have unveiled interesting differences, but also pointed to commonalities throughout the Universe. For example, the oldest stars in our Galaxy lie in the galactic halo, the spherical 'cloud' of thinly scattered globular clusters and old stars surrounding it. What we are seeing is that the abundance pattern of rare heavy elements in halo stars - such as barium, europium and platinum - mirror those in our Solar System.

This is quite surprising, given the billions of years that elapsed between the birth of halo stars and our Sun. It implies that the formative processes for these elements,

including the types of stars and internal conditions, are remarkably robust.

Isotopic studies of certain elements back up this finding. Although it is much more difficult to obtain measurements of individual isotopes of a given element than the abundance of the element itself, recent observations of halo stars indicate that the abundances of europium and barium isotopes have also been consistent with the values we see in our Solar System. And the evidence that this commonality may extend beyond our Galaxy is growing.

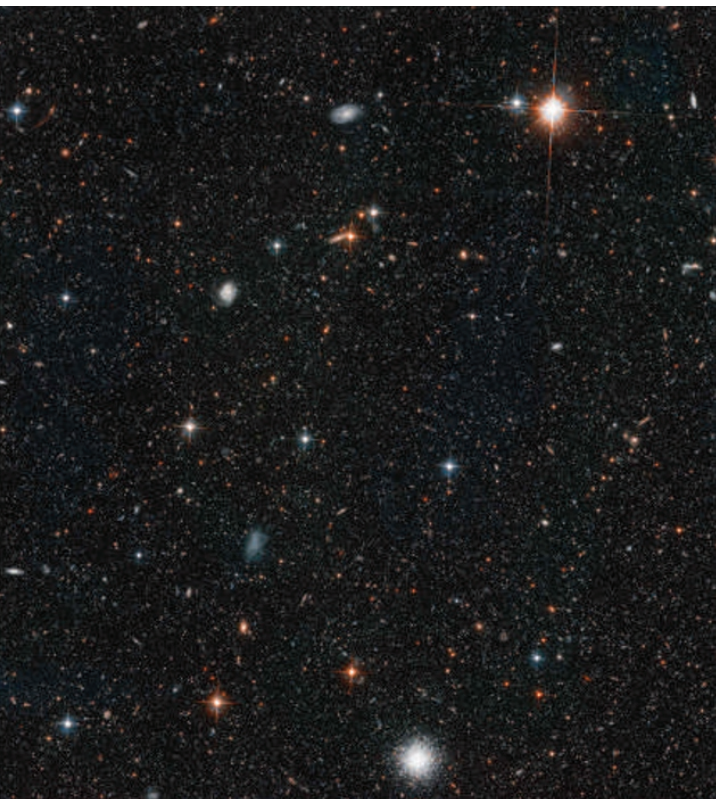

imilarities in stars hold for neighbouring galaxies and beyond.

Studies of dwarf spheroidal galaxies have given us some tantalizing findings. These dim elliptical galaxies have star formation rates and histories distinct from those of the Milky Way, for instance leaving them with different proportions of high- and low-mass stars. Iron-poor stars in four dwarf spheroidal galaxies were observed to have heavy-element abundances similar to those of the Milky Way's halo stars. This is particularly striking at the very lowest and presumably earliest metallicities (or proportions of elements other than hydrogen and helium) where the abundance ratios of certain elements have similar values, and seem to be dominated by one formation process. Elements heavier in mass than iron are synthesized inside stars via nuclear reactions that involve a combination of neutron-capture processes. These processes only occur on two timescales: rapid (much faster than a second) or slow (taking hundreds to thousands of years). Metal-poor stars in both the Milky Way and dwarf spheroidal galaxies are dominated by abundance patterns typical of the rapid process, which is thought to take place in the rare massive stars that explode as supernovae.

Is there any evidence that this commonality in stellar abundance patterns holds beyond our galactic neighbours? Yes. About 25 elemental abundances have been detected in a galaxy almost 12 billion light years away, formed a few billion years after the Big Bang. The number of heavy elements seen in it is relatively modest, but the abundance pattern is again consistent with the kind of relative or scaled abundance distribution we see in the Solar System. This is startling, and a strong indication that the solar abundances are, in many ways, cosmic.

A number of puzzles remain. We know, for example, that there are a few stars in the Milky Way with extremely low iron and extremely low heavy-element abundances. This suggests that some of the very first stars in our Galaxy were incapable of producing any significant fraction of heavy elements. So there may have been several generations of stars that formed early in our Galaxy. If so, how did they differ from each other? And there are certainly differences in iron abundances and star formation rates from galaxy to galaxy.

The abundance patterns we do see suggest more commonalities than differences, with neutron-capture element formation occurring everywhere early in the history of the Universe in a roughly similar manner. A focus on the similarities could give us a more coherent picture of our very interesting Universe.

John Cowan is David Ross Boyd professor in the Homer L. Dodge Department of Physics and Astronomy, University of Oklahoma, Norman, Oklahoma 73019, USA.

\section{FURTHER READING}

Cowan, J. J. \& Sneden, C. Nature 440, 1151 (2006).

Prochaska, J. X., Howk, J. C. \& Wolfe, A. Nature 423, 57-59 (2003)

Shetrone, M. et al. Astron. J. 125, 684-706 (2003). 